\title{
Revue Gouvernance
}

Governance Review

\section{Lettre du rédacteur en chef \\ Letter from the Editor}

\section{Christian Rouillard}

Volume 4, numéro 1, 2007

URI : https://id.erudit.org/iderudit/1039113ar

DOI : https://doi.org/10.7202/1039113ar

Aller au sommaire du numéro

Éditeur(s)

Centre d'études en gouvernance de l'Université d'Ottawa

ISSN

1912-0362 (numérique)

Découvrir la revue

Citer ce document

Rouillard, C. (2007). Lettre du rédacteur en chef. Revue Gouvernance /

Governance Review, 4(1). https://doi.org/10.7202/1039113ar d'utilisation que vous pouvez consulter en ligne.

https://apropos.erudit.org/fr/usagers/politique-dutilisation/ 


\title{
Lettre du rédacteur en chef
}

\author{
Vol.4, No. 1, mai 2007, Page 1
}

\section{Christian Rouillard}

Ce nouveau numéro de Revue gouvernance offre quatre textes qui couvrent des sujets aussi variés que les partenariats public-privé (PPP), le rôle des comités d'audit sur les états financiers d'une firme privée, les implications épistémologiques et ontologiques d'une théorie de l'énoncé pour la recherche sur la gouvernance et, enfin, les réformes des systèmes de santé nationalisés au Québec et au Royaume-Uni.

Dans le premier texte, Christopher Stoney (Carleton University) et Robert Hilton (Gouvernement du Canada) se penche sur l'expérience du train « Light Rail Transit », le projet le plus important, en termes financiers, de l'histoire de la ville d'Ottawa. Impliquant non seulement l'administration municipale de la capitale fédérale et une firme privée transnationale, mais aussi les gouvernements fédéral et ontarien, ce projet avorté à grand frais est une illustration de la complexité des mégaprojets en mode PPP impliquant des ententes financières trigouvernementales, tout autant que de la dynamique politique qui s'y greffe invariablement. L'analyse critique de Stoney et Hilton enrichit considérablement le débat public sur cette question d'une actualité brûlante.

Dans le deuxième texte, Mohamed Ali Zarai et Wided Bettabai (Institut supérieur de gestion de Tunis) étudient l'impact des comités d'audit sur la qualité des états financiers de firmes privées américaines. Privilégiant une analyse quantitative de données empiriques d'un échantillon de cent firmes privées de différents secteurs d'activités aux Etats-Unis durant la période 2000-2004, les auteurs parviennent à identifier certains des principaux facteurs explicatifs de l'impact positif des comités d'audit sur les états financiers, un enjeu central pour la gouvernance d'entreprise. Par-delà les seules firmes privées, la discussion des résultats est d'un intérêt manifeste pour les organisations publiques, particulièrement pour les sociétés d'État et les entreprises publiques.

Dans le troisième texte, Benaissa Jebbour (LIPSOR, CNAM de Paris) offre une réflexion théorique sur la pertinence et l'intérêt d'une théorie de l'énoncé pour les études managérielles et politiques, notamment eu égard aux différentes questions que soulève la gouvernance organisationnelle. D'une sensibilité poststructuraliste, cette recherche théorique permet d'établir une complémentarité nouvelle entre deux traditions philosophiques qui tendent habituellement à s'ignorer mutuellement, à savoir : la phénoménologie herméneutique et la philosophie analytique de l'action.

Dans le quatrième texte, Mélanie Bourque (Université du Québec en Outaouais) s’intéresse aux réformes administratives qu'ont connues aux cours des vingt dernières années les systèmes de santé nationalisés au Québec et au Royaume-Uni. À partir d'une analyse néo-institutionnaliste, elle fait ressortir la nature et l'ampleur des différentes contraintes qui se sont respectivement imposées à ces deux ambitieuses réformes. Par-delà son intérêt académique, la recherche de Bourque nourrit directement le débat public, toujours en cours, au sujet du rôle du privé dans le système de santé québécois.

Bonne lecture! 